\title{
Assessment of Hepatic and Lipid Profiles Following 12 Weeks of Aerobic Exercise in Overweight Postmenopausal Women
}

\author{
Bakhtyar Tartibian $^{1 *}$, Abbas Malandish ${ }^{2 *}$, Roghaiyeh Afsargharehbagh ${ }^{3}$, Rasoul Eslami ${ }^{4}$, Zeinab Sheikhlou ${ }^{2}$ \\ 'Department of Sports Injuries and Corrective Exercises, Faculty of Physical Education \& Sport Sciences, Allameh Tabataba'i \\ University, Tehran, Iran. \\ ${ }^{2}$ Department of Exercise Physiology, Faculty of Sport Sciences, Urmia University, Urmia, Iran \\ ${ }^{3}$ Department of Interventional Cardiology, Shohada Hospital, Urmia University of Medical Sciences, Urmia, Iran. \\ ${ }^{4}$ Department of Sport Injuries \& Corrective Exercises, Faculty of Physical Education \& Sport Sciences, Allameh Tabataba'i \\ University, Tehran, Iran
}

\section{*Correspondence to Bakhtyar Tartibian, Tel: +989126090551 Email: ba.tartibian@gmail.com Abbas Malandish, Tel: +989143922878 Email: malandish@gmail.com}

Received June 21, 2018 Accepted September 8, 2018 Published online December 31 2018
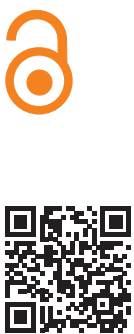

Please cite this article as follows: Tartibian B, Malandish A, Afsargharehbagh R, Eslami R, Sheikhlou Z. Assessment of hepatic and lipid profiles following 12 weeks of aerobic exercise in overweight postmenopausal women . Int J Basic Sci Med. 2018;3(4):159167. doi:10.15171/ ijbms.2018.28.

\begin{abstract}
Introduction: The impact of aerobic training on liver function by modulating hepatic enzymes and lipid profiles in overweight women is uncertain. The aim of our study was to examine the impact of 12-week aerobic exercise training on hepatic and lipid profiles and cardiorespiratory indices in overweight women aged over 50 years.

Methods: Thirty sedentary and overweight postmenopausal women (PMW) over 50 years old were randomly divided into 2 groups: exercise $(E x, n=15)$ and control $(C, n=15)$ groups. The Ex group performed moderate-intensity aerobic exercise $(60 \mathrm{~min} / \mathrm{d}, 3$ days/week at $65 \%-70 \%$ of maximal heart rate reserve $\left.\left[\mathrm{HR}_{\max }\right]\right)$ for 12 weeks. The $\mathrm{C}$ group participated in no intervention during a 3-month period and maintained their normal daily lifestyle. The serum levels of hepatic and lipid profiles were assessed at baseline and after week 12. Descriptive and inferential (ANCOVA test) statistics were used to analyze the data using SPSS software version 23.0 at a significance level of $P<0.05$.

Results: After 12 weeks of exercise intervention, the serum levels of hepatic and lipid profiles were not significantly different in the Ex group compared to the $C$ group $(P>0.05)$. However, maximal oxygen uptake $\left(\mathrm{VO}_{2 \max }\right)$, walking-jogging time to exhaustion (WJTE), and alkaline phosphatase (ALP) significantly increased in the Ex group $(P<0.05)$. In contrast, systolic blood pressure $(\mathrm{SBP})$ significantly decreased in the Ex group $(P<0.05)$.

Conclusion: The results demonstrated that 12-week moderate-intensity aerobic exercise (jogging and walking) at $65 \%-70 \%$ of $\mathrm{HR}_{\max }$ did not affect the liver function without modulating hepatic enzymes and lipid profiles in overweight women over 50 years old, whereas cardiorespiratory fitness (CRF) by modulating VO2max, WJTE, and SBP was improved.

Keywords: Aerobic exercise intervention, Liver enzymes, Lipid profiles, Overweight, Menopause.
\end{abstract}

\section{Introduction}

Obesity and overweight, increased triglycerides (TGs), high-density lipoprotein cholesterol (HDL) reduction, and hypertension are associated with an increase in serum levels of hepatic indices and the risk of developing metabolic syndrome. ${ }^{1-5}$ Most studies have shown that liver function indices, especially serum levels of hepatic enzymes and lipid profiles were closely correlated with metabolic syndrome. It has been estimated that metabolic syndrome such as liver dysfunction along with the epidemic of obesity, will be the main reasons of liverassociated morbidity and mortality by $2030 .^{3}$ The researches indicate that serum levels of hepatic enzymes ${ }^{6-9}$ and lipid profiles ${ }^{10}$ are reliablefunctionalindices for assessing hepatic function. Aspartate aminotransferase (AST), alanine aminotransferase (ALT), alkaline phosphatase (ALP), lactate dehydrogenase (LDH), $\gamma$-glutamyl transferase (GGT), and 5 '-nucleotidase are liver enzymes. ${ }^{6}$

AST is a dimeric enzyme with a molecular

(c) 2018 The Author(s); Published by Zabol University of Medical Sciences. This is an open-access article distributed under the terms of the Creative Commons Attribution License (http://creativecommons.org/licenses/by/4.0), which permits unrestricted use, distribution, and reproduction in any medium, provided the original work is properly cited. 
weight of $45 \mathrm{kDa}$ and 400 amino acids which is found mainly in the liver. ${ }^{7}$ The normal serum levels of AST are less than 34 and $52 \mathrm{U} / \mathrm{L}$ in women and men, respectively. AST level has a direct correlation with the number of damaged hepatocytes, however, its serum concentration also increases during the heart attack and muscle damage. ${ }^{711}$ ALT is a non-glycosylated polypeptide singlechain cytoplasmic enzyme with a molecular weight of $54.5 \mathrm{kDa}$ and 496 amino acids which is found mainly in the liver. ${ }^{12}$ ALT catalyzes transmutation of glutamate and pyruvate into alpha-ketoglutarate and L-alanine. The normal serum levels of ALT are similar to the AST. Circulating ALT is catabolized in the liver (half-life of ALT is $47 \pm 10$ hours which is longer than that of AST; $17 \pm 5$ hours). ${ }^{12}$ It seems that increased ALT can be a sign of damage in the hepatocytes and/or cardiac or skeletal muscle necrosis., ${ }^{71,12}$ ALP is a tetrameric glycoprotein enzyme with a molecular weight of $140 \mathrm{kDa}$ which is mainly secreted by the liver and bone marrow. ${ }^{13}$ The normal level of ALP in adults is $145 \mathrm{U} / \mathrm{L}$ with increasing levels during menopause. ${ }^{13}$ Increased serum ALP has been associated with liver and bone diseases. ${ }^{13}$

TGs are ester compounds of glycerol and fatty acids with 2 endogenous and exogenous forms, the endogenous form of which is produced in the liver and is transported by very low-density lipoprotein (VLDL) and its exogenous form is produced in mucosal cells of the intestine. TGs as the key factor in VLDL and chylomicrons play an important role in metabolism. The normal level of TGs is up to $150 \mathrm{mg} / \mathrm{dL}$ and its higher values are associated with the liver diseases. ${ }^{14}$ Cholesterol is an extremely important biological molecule in the blood, liver, brain, kidneys, and nervous fibers that has roles in membrane structure as well as in the synthesis of steroid hormones, bile acids, and vitamin D. Low cholesterol levels are related to the liver diseases as well as acute heart attack. LDL is composed of $50 \%$ cholesterol, $20 \%$ phospholipid and a small amount of TGs that play a role in the transfer of cholesterol into tissues. ${ }^{7,10} \mathrm{HDL}$, as smallest lipoprotein particles, is involved in reverse cholesterol transport (RCT) by the liver. The normal level of HDL is $40-60 \mathrm{mg} /$ $\mathrm{dL}$ in adults. High HDL levels have a protective effect on the liver and cardiovascular system (CVS), while its low levels increase the risk of heart and liver diseases. ${ }^{14}$

Multiple studies have reported that aerobic exercise, along with weight loss and appropriate lifestyle, can improve liver function and prevent liver diseases. ${ }^{15,16}$ However, many studies have reported effects of aerobic training on the hepatic and lipid profiles, but their results are contradictory.,17-19 On the other hand, in aged individuals and especially in postmenopausal women (PMWs), the accumulation of visceral fat due to a reduction in sex hormones such as estrogen and progesterone leads to an increase in CVS, metabolic and liver diseases. ${ }^{13}$ In other words, in the menopause period, the relationships between estrogen reduction and biochemical effects due to aging process results in the hepatic damage. ${ }^{20}$ Moreover, some studies have reported that liver function is decreased around $1 \%$ per year after 40 to 50 years of age $\mathrm{e}^{20}$ and it reaches up to $40 \%$ in old age and elderly, especially in women. ${ }^{21}$

Furthermore, $21 \%$ to $43 \%$ of Iranian people suffer from liver dysfunction. ${ }^{17}$ Regarding the beneficial role of aerobic exercise, we here assessed its potential effects on the improvement of liver function in PMWs. Here, we examined the effect of 12 weeks of warm up- walking and jogging moderate intensity aerobic exercise training program- recovery (W-WJMIAEP-R) on physiological indices, hepatic enzymes and lipid profiles in PMWs.

\section{Materials and Methods}

Subjects

This study was a randomized controlled trial which was conducted in April 2018 at Allameh Tabataba'I University, Iran. The statistical population included all overweight and sedentary PMWs aged over 50 years in Urmia, Iran. During the public notice of exercise and PMWs, 200 overweight and sedentary PMWs declared, among whom 30 volunteers qualified for the study. The sample size was calculated using $G^{*}$ Power software ${ }^{22}$ (version 8.0.50727.42; Franz Faul, Universitat Kiel, Dusseldorf, Germany) for univariate analysis of variance, which revealed that 15 participants per group were needed.

\section{Inclusion Criteria}

The inclusion criteria included PMWs with the age of $>$ 50 years old, spending between 1 to 5 years at menopause, which was controlled by evaluating the serum levels of $17 \beta$-estradiol (11-65 $\mu \mathrm{L} / \mathrm{pg}$ ) and progesterone (0.1-1 $\mu \mathrm{L} / \mathrm{ng}$ ), body mass index (BMI) $>25 \mathrm{~kg} / \mathrm{m}^{2}$, no history of current diseases affecting hepatic metabolism as well as any clinical problem in the liver and CVS, and no history of regular exercises, and recent smoking. The CVS function was evaluated based on electrocardiogram (ECG) (Esaote Spa, Firenze, Italy).

\section{Exclusion Criteria}

Exclusion criteria included the diagnosis of chronic or any recent diseases affecting liver metabolism, discontinuing of regular exercise protocol during the study, having a special dietary regimen, and receiving any pharmacological treatments during the study.

\section{Allocation into Experimental Groups}

Health and physical activity level of PMWs were measured by sports medicine and self-report questionnaires. ${ }^{23}$ At a meeting, all participants met the stages of the implementation of the study, and all of them received written consent. The subjects were then randomly assigned to the $\operatorname{Ex}(n=15)$ or $C(n=15)$ groups by simple randomization method. 


\section{Sample Size}

The sample size was calculated using $G^{*}$ Power software for univariate analysis of variance for detecting a medium effect size (Cohen d 1/4 0.4) with an alpha of 0.05 and power of $95 \%$. The sample size was calculated as 15 participants per group.

\section{Dietary Assessment}

Dietary data were assessed via a 3-day food record ${ }^{24}$ in the first and last week of the study. The participants were requested to maintain their normal diet during 12 weeks. Information on the use of medications/ supplements was also obtained via standard and selfreported questionnaires. ${ }^{24}$ Nutritional and dietary data were analyzed by nutrition analysis software (Nutrition data pro $^{\mathrm{TM}}$ v1.1, StarApps Company, Washington, USA).

\section{Exercise Program}

The graded exercise test (GXT) on treadmill by George et al was used to determine maximal oxygen uptake $\left(\mathrm{VO}_{2 \max }\right) .^{25}$ By using the Borg scale, rating of perceived exertion (RPE) was recorded in the last 10 seconds of each stage. Two of the following four criteria were required for a test to be considered maximal: (1) a plateau in $\mathrm{VO}_{2}$ despite increasing workload; (2) respiratory exchange ratio $($ RER) $\geq 1.10$; (3) maximal heart rate within 10 beats of age predicted $\max [\max H R=(208-(0.7 \times$ age in year $)]$; and (4) $\mathrm{RPE} \geq 17$. The Ex group performed 12 weeks of W-WJMIAEP-R protocol with $65 \%$ to $70 \% \mathrm{HR}_{\max }$ of training on a treadmill, 3 sessions per week and 50 to 60 minutes per session (180-200 minutes in a week) in the morning. Each training session included 10 minutes of warming up, 40 minutes of walking and jogging aerobic exercise, and 10 minutes of cooling down/recovery. The exercises were performed with maximum heart rate $\left(\mathrm{HR}_{\max }\right)$ of $50 \%$ (the first week), 60\% (the next 2 weeks), $65 \%$ (the following 4 weeks), and 70\% (the last 5 weeks). ${ }^{25}$ All exercise sessions were performed between 08:00 and 10:00 AM and the participants were trained by exercise physiologist. The $\mathrm{C}$ group participated in no intervention and continued their normal daily lifestyle and dietary habits during 12 weeks.

\section{Laboratory Measurements}

\section{Blood Sampling and Assays}

The serum levels of sex hormones (17 $\beta$-estradiol and progesterone), hepatic enzymes (AST, ALT, and ALP), and lipid profiles (TGs, total cholesterol [TC], HDL, and LDL) were measured at baseline ( 24 hours before initiation of the study) and 24 hours after the last session of training. After 12-hour overnight fasting, blood samples (5 mL) were taken in the early morning (between 07:00 and 08:00 AM). To separate serum, blood samples were centrifuged at $3000 \mathrm{rpm}$, after 15-minute clotting at room temperature, for 15 minutes at $4^{\circ} \mathrm{C}$ by centrifuge machine. Serum levels of $17 \beta$-estradiol (1561-DRG, Euroimmun, Germany) and progesterone (2633-DRG, Euroimmun, Germany) were measured using enzyme-linked immunosorbent assay (ELISA) machine (Stat Fax 4200- Awareness Technology, USA). Serum levels of AST, ALT, ALP, TGs, TC, HDL, and LDL were measured by standard automated techniques using specific kits (BT-1500, AutoAnalyzer, Italy).

\section{Data Analysis}

All the data were expressed as mean \pm standard deviation (SD). Kolmogorov-Smirnov test and Levene test were used for normality of the data. Between-group differences were determined by independent samples $t$ test at baseline and post-exercise. In addition, univariate analysis of variance (ANCOVA) test in a general linear model was applied to determine the changes of all variables between the 2 groups during the time (intervention - time interaction). The difference between baseline and post-exercise values for each group was investigated using paired samples $t$ test. The correlations between hepatic enzymes and lipid profiles among PMWs at baseline and post-exercise were used by Pearson's correlation coefficient test. The statistical software program SPSS (SPSS Co, Chicago IL, version 23) for windows was applied to analyze the data. The statistical significance was considered as 2 tailed $P<0.05$ for all tests.

\section{Results}

Baseline characteristics of participants did not show a significant difference between groups regarding physiological indices, cardiorespiratory fitness (CRF), menopause status, hepatic enzymes, and lipid profiles $(P>0.05)$, except for ALP and TC $(P<0.05)$ (Table 1$)$.

Following 12 weeks of W-WJMIAEP-R intervention, systolic blood pressure (SBP) significantly decreased in the Ex group while $\mathrm{VO}_{2 \max }$, walking-jogging time to exhaustion (WJTE), and ALP showed a significant increase $(P<0.05)$. In addition, $17 \beta$-estradiol and progesterone levels significantly decreased while TC and LDL showed a significant increase in the $\mathrm{C}$ group (Table 1). The effect size (Eta) coefficient showed $53.5 \%$ and $55.2 \%$ increase in $\mathrm{VO}_{2 \max }$ and WJTE, respectively in the EX group compared to the $\mathrm{C}$ group, whereas other factors did not show considerable changes (Table 1).

Dietary intake status in total energy intake (TEI), fat, carbohydrate $(\mathrm{CHO})$, and protein did not show any significant differences in within/between group analyses among PMWs at baseline and post-exercise (Table 2).

Table 3 shows that the correlations of ALP with TC and LDL were significantly positive in the Ex group at baseline and week 12. Moreover, the correlations of AST with TC and LDL were significantly positive in the $\mathrm{C}$ group at week 12 .

\section{Discussion}

The aim of this study was to examine the impact of 12 week W-WJMIEP-R intervention on hepatic enzymes 
Table 1. Physiological Indices, Menopause Status, and Liver Parameters of Postmenopausal Women in 2 Study Groups (Aerobic Exercise and Control) at Baseline and Week 12

\begin{tabular}{|c|c|c|c|c|c|}
\hline Variables & Exercise $(n=15)$ & Control $(n=15)$ & $P^{b}$ Value & $P^{c}$ Value & Eta Coefficient $(\%)$ \\
\hline \multicolumn{6}{|c|}{ Physiological Indices } \\
\hline \multicolumn{6}{|l|}{ Age $(y)$} \\
\hline Baseline & $52.20 \pm 4.39$ & $52.21 \pm 3.62$ & ns & \multirow{3}{*}{ ns } & \multirow{2}{*}{ ns } \\
\hline Week 12 & $52.20 \pm 4.39$ & $52.21 \pm 3.62$ & ns & & \\
\hline$P^{a}$ value & ns & ns & & & \\
\hline \multicolumn{6}{|c|}{ Height (cm) } \\
\hline Baseline & $156.93 \pm 5.39$ & $158.21 \pm 4.88$ & ns & \multirow{2}{*}{ ns } & \multirow{2}{*}{ ns } \\
\hline Week 12 & $156.93 \pm 5.39$ & $158.21 \pm 4.88$ & ns & & \\
\hline$P^{a}$ value & ns & ns & & & \\
\hline \multicolumn{6}{|c|}{ Weight (kg) } \\
\hline Baseline & $71.26 \pm 10.04$ & $75.71 \pm 14.76$ & 0.348 & \multirow{3}{*}{0.390} & \multirow{3}{*}{$0.019(1.9 \%)$} \\
\hline Week 12 & $71.06 \pm 9.91$ & $75.42 \pm 13.55$ & 0.329 & & \\
\hline$P^{a}$ value & 0.757 & 0.959 & & & \\
\hline \multicolumn{6}{|c|}{ BMI $\left(\mathrm{kg} / \mathrm{m}^{2}\right)$} \\
\hline Baseline & $29.03 \pm 4.70$ & $30.18 \pm 5.78$ & 0.560 & \multirow{3}{*}{0.974} & \multirow{3}{*}{$0.001(0.1 \%)$} \\
\hline Week 12 & $29.62 \pm 4.61$ & $30.00 \pm 5.03$ & 0.850 & & \\
\hline$P^{a}$ value & 0.160 & 0.919 & & & \\
\hline \multicolumn{6}{|c|}{ Resting heart rate (beats/min) } \\
\hline Baseline & $80.80 \pm 9.53$ & $76.57 \pm 11.19$ & 0.282 & \multirow{3}{*}{0.556} & \multirow{3}{*}{$0.014(1.4 \%)$} \\
\hline Week 12 & $80.06 \pm 11.57$ & $76.21 \pm 10.13$ & 0.350 & & \\
\hline$P^{a}$ value & 0.825 & 0.913 & & & \\
\hline \multicolumn{6}{|c|}{ Systolic blood pressure (mm Hg) } \\
\hline Baseline & $128.46 \pm 22.01$ & $125.07 \pm 20.54$ & 0.672 & \multirow{3}{*}{0.226} & \multirow{3}{*}{$0.048(4.8 \%)$} \\
\hline Week 12 & $116.66 \pm 14.27$ & $121.21 \pm 14.39$ & 0.401 & & \\
\hline$P^{a}$ value & $0.026^{*}$ & 0.503 & & & \\
\hline \multicolumn{6}{|c|}{ Diastolic blood pressure (mm Hg) } \\
\hline Baseline & $77.53 \pm 14.73$ & $74.35 \pm 11.07$ & 0.520 & 0907 & 0 \\
\hline Week 12 & $71.33 \pm 10.66$ & $70.42 \pm 7.66$ & 0.796 & 0.907 & $0.001(0.1 \%)$ \\
\hline$P^{a}$ value & 0.124 & 0.312 & & & \\
\hline & & piratory Fitness In & & & \\
\hline Maximal o & $/ \mathrm{kg} / \mathrm{min})$ & & & & \\
\hline Baseline & $39.10 \pm 2.30$ & $40.12 \pm 5.70$ & 0.715 & $0.002^{\ddagger}$ & $0.535(53.5 \%)$ \\
\hline Week 12 & $43.40 \pm 2.20$ & $39.25 \pm 6.40$ & 0.175 & & \\
\hline$P^{a}$ value & $0.001^{*}$ & 0.133 & & & \\
\hline Walking \& & $(\min )$ & & & & \\
\hline Baseline & $9.95 \pm 2.60$ & $9.75 \pm 2.50$ & 0.895 & $0001^{\neq}$ & $0552(552 \%)$ \\
\hline Week 12 & $12.15 \pm 2.00$ & $9.63 \pm 2.65$ & $0.026 t$ & $0.001^{7}$ & $0.552(55.2 \%)$ \\
\hline$P^{a}$ value & $0.001^{*}$ & 0.469 & & & \\
\hline & & enopause Status & & & \\
\hline Menopaus & & & & & \\
\hline Baseline & $3.20 \pm 1.25$ & $2.65 \pm 1.20$ & ns & & \\
\hline Week 12 & $3.20 \pm 1.25$ & $2.65 \pm 1.20$ & ns & ns & ns \\
\hline$P^{a}$ value & ns & ns & & & \\
\hline $17 \beta$-estrad & & & & & \\
\hline Baseline & $31.53 \pm 16.28$ & $41.06 \pm 12.65$ & 0.202 & 0123 & 0117 (11 7\%) \\
\hline Week 12 & $29.73 \pm 15.36$ & $23.85 \pm 12.30$ & 0.402 & 0.123 & $0.117(11.7 \%)$ \\
\hline$P^{a}$ value & 0.693 & $0.031^{*}$ & & & \\
\hline Progestero & & & & & \\
\hline Baseline & $0.18 \pm 0.10$ & $0.30 \pm 0.14$ & 0.084 & 0495 & $0026(26 \%)$ \\
\hline Week 12 & $0.14 \pm 0.07$ & $0.20 \pm 0.17$ & 0.401 & 0.495 & $0.026(2.6 \%)$ \\
\hline$P^{a}$ value & 0.104 & $0.033^{*}$ & & & \\
\hline
\end{tabular}


Table 1. Continued

\begin{tabular}{|c|c|c|c|c|c|}
\hline Variables & Exercise $(n=15)$ & Control $(n=15)$ & $P^{\mathrm{b}}$ Value & $P^{c}$ Value & Eta Coefficient (\%) \\
\hline \multicolumn{6}{|c|}{ Hepatic Enzymes } \\
\hline \multicolumn{6}{|l|}{ AST (IU/L) } \\
\hline Baseline & $17.33 \pm 7.11$ & $19.21 \pm 5.01$ & 0.421 & \multirow{2}{*}{0.419} & \multirow{2}{*}{$0.025(2.5 \%)$} \\
\hline Week 12 & $23.40 \pm 7.58$ & $20.71 \pm 6.56$ & 0.319 & & \\
\hline$P$ a value & 0.058 & 0.532 & & & \\
\hline \multicolumn{6}{|l|}{ ALT (IU/L) } \\
\hline Baseline & $9.33 \pm 7.11$ & $10.14 \pm 3.75$ & 0.418 & \multirow{2}{*}{0.382} & \multirow{2}{*}{$0.048(4.8 \%)$} \\
\hline Week 12 & $13.00 \pm 11.38$ & $10.35 \pm 3.95$ & 0.672 & & \\
\hline$P^{a}$ value & 0.201 & 0.894 & & & \\
\hline \multicolumn{6}{|l|}{ ALP (IU/L) } \\
\hline Baseline & $181.33 \pm 57.87$ & $227.85 \pm 47.18$ & $0.026+$ & \multirow{2}{*}{0.941} & \multirow{2}{*}{$0.001(0.1 \%)$} \\
\hline Week 12 & $213.60 \pm 59.53$ & $227.92 \pm 50.91$ & 0.494 & & \\
\hline$P^{a}$ value & $0.033^{*}$ & 0.997 & & & \\
\hline \multicolumn{6}{|c|}{ Lipid Profiles } \\
\hline \multicolumn{6}{|l|}{ TGs $(\mu / \mathrm{L})$} \\
\hline Baseline & $182.20 \pm 81.56$ & $144.85 \pm 71.51$ & 0.202 & \multirow{2}{*}{0.978} & \multirow{2}{*}{$0.001(0.1 \%)$} \\
\hline Week 12 & $172.33 \pm 77.10$ & $157.85 \pm 66.22$ & 0.593 & & \\
\hline$P^{a}$ value & 0.608 & 0.580 & & & \\
\hline \multicolumn{6}{|l|}{$\mathrm{TC}(\mu / \mathrm{L})$} \\
\hline Baseline & $243.53 \pm 24.62$ & $218.07 \pm 29.41$ & $0.017 \dagger$ & \multirow{2}{*}{0.525} & \multirow{2}{*}{$0.016(1.6 \%)$} \\
\hline Week 12 & $252.53 \pm 29.18$ & $240.50 \pm 35.49$ & 0.326 & & \\
\hline$P^{a}$ value & 0.174 & $0.011 *$ & & & \\
\hline \multicolumn{6}{|l|}{$\operatorname{HDL}(\mu / \mathrm{L})$} \\
\hline Baseline & $34.60 \pm 6.62$ & $35.57 \pm 6.72$ & 0.698 & \multirow{3}{*}{0.900} & \multirow{3}{*}{$0.001(0.1 \%)$} \\
\hline Week 12 & $34.66 \pm 6.27$ & $35.50 \pm 6.51$ & 0.728 & & \\
\hline$P^{a}$ value & 0.963 & 0.962 & & & \\
\hline \multicolumn{6}{|l|}{$\operatorname{LDL}(\mu / \mathrm{L})$} \\
\hline Baseline & $172.00 \pm 19.35$ & $152.07 \pm 35.32$ & 0.068 & \multirow{2}{*}{0.840} & \multirow{2}{*}{$0.002(0.2 \%)$} \\
\hline Week 12 & $173.20 \pm 23.09$ & $173.07 \pm 35.02$ & 0.363 & & \\
\hline$P^{a}$ value & 0.883 & $0.038^{*}$ & & & \\
\hline
\end{tabular}

Abbreviations: BMI, body mass index; TGs, triglycerides; TC, total cholesterol; ALP, alkaline phosphatase; ALT, alanine aminotransferase; AST, aspartate aminotransferase; HDL, high density lipoprotein; LDL, Low density lipoprotein.

Values are expressed as mean $\pm \mathrm{SD}$.

$* P^{a}<0.05$, significant difference from baseline values by paired samples $t$ test (within groups analysis).

$+P^{\mathrm{b}}<0.05$, significant difference in between-groups by independent samples $t$ test,

$\mp P^{c}<0.05$, significantly different between groups analyzed by univariate analysis of variance (ANCOVA) (exercise and time interaction).

Eta $=$ Partial Eta squared or estimates of effect size.

and lipid profiles in overweight PMWs over 50 years old. Our study indicated that 12-week W-WJMIEP-R intervention improved CRF through positive adaptations in physiological indices including an increase in the $\mathrm{VO}_{2 \max }$ and WJTE among overweight PMWs while the other variables did not show considerable changes during the time (group - time interaction). Furthermore, we observed increased ALP and decreased SBP in the exercise group and decreased $17 \beta$-estradiol and progesterone and increased TC and LDL in the C group after 12 weeks of W-WJMIEP-R. The findings of our study were similar to the results of some previous studies ${ }^{26-28}$ and were inconsistent with others. ${ }^{19,29,30}$ Hosseinikakhk et $\mathrm{al}^{26}$ reported that the hepatic enzymes including AST and ALT did not change after 8 -week resistance training at $50 \%$ to $70 \% \mathrm{HR}_{\max }$ reserve in men with liver diseases which was consistent with the findings of the current study. In addition, Mohammadrahimi et $\mathrm{al}^{27}$ reported that 12 weeks of aerobic exercise at $50 \%$ to $70 \% \mathrm{HR}_{\max }$ had no significant effect on the AST and ALT in overweight PMWs.

The findings of this study were consistent with those studies with aspects similar to our protocol and subjects including Mohammadrahimi et $\mathrm{al}^{27}$ (12 weeks of aerobic training at $50 \%-70 \% \mathrm{HR}_{\max }$, three sessions per week, and 50-60 minutes per training session in overweight PMWs), Hosseinikakhk et $\mathrm{al}^{26}$ (similar exercise intensity at $50 \%-70 \% \mathrm{HR}_{\max }$ ), and Sadeghi et $\mathrm{al}^{31}$ (the same exercise protocol of 12 weeks aerobic exercise and similar subjects of obese women). In contrast, the difference in the type of training protocol of resistance exercise in a study by Slentz et $\mathrm{al}^{29}$ and short-term aerobic training protocol (6 
Table 2. Differences in Dietary Intake of Postmenopausal Women at Baseline and After 12 weeks of Aerobic Exercise Intervention

\begin{tabular}{|c|c|c|c|c|}
\hline Variables & Exercise $(n=15)$ & Control $(n=15)$ & Pb Value & $P^{c}$ Value \\
\hline \multicolumn{5}{|c|}{ Total energy intake (TEI) (kcal/d) } \\
\hline Baseline & $2245.85 \pm 545.81$ & $2365.12 \pm 515.62$ & 0.602 & \multirow{3}{*}{0.341} \\
\hline Week 12 & $2305.35 \pm 335.25$ & $2299.25 \pm 312.60$ & 0.966 & \\
\hline$P$ a value & 0.557 & 0.419 & & \\
\hline \multicolumn{5}{|l|}{ Fat $(\mathrm{g})$} \\
\hline Baseline & $103.10 \pm 30.12$ & $104.20 \pm 32.15$ & 0.936 & \multirow{3}{*}{0.645} \\
\hline Week 12 & $105.15 \pm 28.10$ & $101.30 \pm 21.15$ & 0.788 & \\
\hline$P^{a}$ value & 0.996 & 0.568 & & \\
\hline \multicolumn{5}{|c|}{ Carbohydrate $(\mathrm{CHO})(\mathrm{g})$} \\
\hline Baseline & $255.22 \pm 114.10$ & $295.99 \pm 78.50$ & 0.345 & \multirow{3}{*}{0.390} \\
\hline Week 12 & $265.75 \pm 85.25$ & $284.20 \pm 65.75$ & 0.603 & \\
\hline$P^{a}$ value & 0.435 & 0.202 & & \\
\hline \multicolumn{5}{|l|}{ Protein (g) } \\
\hline Baseline & $89.50 \pm 23.30$ & $79.75 \pm 25.60$ & 0.334 & \multirow{3}{*}{0.474} \\
\hline Week 12 & $91.00 \pm 21.50$ & $80.20 \pm 23.30$ & 0.245 & \\
\hline$P^{a}$ value & 0.694 & 0.655 & & \\
\hline
\end{tabular}

Values are expressed as mean \pm SD. ${ }^{*} P^{\mathrm{a}}<0.05$, significant difference from baseline values by paired samples $t$ test (within groups analysis). ${ }^{\dagger} P^{b}<0.05$, significant difference baseline and post-intervention values by independent samples $t$ test (between groups analysis). $\neq P^{c}<0.05$, significantly different between groups by univariate analysis of variance (ANCOVA) (exercise-time interaction).

Table 3. The Correlation Between Hepatic Enzymes and Lipid Profiles Among Postmenopausal Women at Baseline and After 12 Weeks of Aerobic Exercise Intervention

\begin{tabular}{|c|c|c|c|c|c|c|c|c|c|c|c|c|}
\hline \multirow{3}{*}{ Variable } & \multicolumn{4}{|c|}{ AST $(\mu / L)$} & \multicolumn{4}{|c|}{$\operatorname{ALT}(\mu / L)$} & \multicolumn{4}{|c|}{$\operatorname{ALP}(\mu / L)$} \\
\hline & \multicolumn{2}{|c|}{ Baseline } & \multicolumn{2}{|c|}{ Week 12} & \multicolumn{2}{|c|}{ Baseline } & \multicolumn{2}{|c|}{ Week 12} & \multicolumn{2}{|c|}{ Baseline } & \multicolumn{2}{|c|}{ Week 12} \\
\hline & $R$ & $\boldsymbol{P}$ & $\boldsymbol{R}$ & $\boldsymbol{P}$ & $R$ & $\boldsymbol{P}$ & $\boldsymbol{R}$ & $\boldsymbol{P}$ & $R$ & $\boldsymbol{P}$ & $R$ & $\boldsymbol{P}$ \\
\hline \multicolumn{13}{|c|}{ Ex Group ( $n=15)$} \\
\hline TGs $(\mathrm{u} / \mathrm{L})$ & -0.50 & 0.05 & 0.12 & 0.65 & -0.24 & 0.37 & 0.27 & 0.31 & 0.00 & 0.97 & 0.16 & 0.56 \\
\hline $\mathrm{TC}(\mathrm{u} / \mathrm{L})$ & -0.26 & 0.33 & 0.41 & 0.12 & -0.19 & 0.49 & 0.49 & 0.05 & 0.52 & $0.04 *$ & 0.57 & $0.02 *$ \\
\hline HDL (u/L) & 0.25 & 0.35 & -0.21 & 0.44 & -0.35 & 0.19 & -0.17 & 0.53 & 0.00 & 0.00 & -0.05 & 0.85 \\
\hline $\mathrm{LDL}(\mathrm{u} / \mathrm{L})$ & 0.00 & 0.99 & 0.49 & 0.05 & -0.14 & 0.60 & 0.49 & 0.06 & 0.65 & $0.00^{*}$ & 0.63 & $0.01 *$ \\
\hline \multicolumn{13}{|c|}{ C Group $(n=15)$} \\
\hline TGs $(\mathrm{u} / \mathrm{L})$ & -0.10 & 0.71 & 0.07 & 0.81 & -0.11 & 0.68 & 0.19 & 0.49 & 0.41 & 0.14 & 0.18 & 0.51 \\
\hline $\mathrm{TC}(\mathrm{u} / \mathrm{L})$ & 0.39 & 0.19 & 0.64 & $0.01 *$ & 0.26 & 0.36 & 0.44 & 0.10 & 0.00 & 0.97 & 0.14 & 0.61 \\
\hline $\mathrm{HDL}(\mathrm{u} / \mathrm{L})$ & -0.17 & 0.54 & 0.00 & 0.00 & -0.07 & 0.77 & -0.10 & 0.71 & 0.20 & 0.48 & 0.34 & 0.23 \\
\hline $\mathrm{LDL}(\mathrm{u} / \mathrm{L})$ & 0.39 & 0.16 & 0.59 & $0.02 *$ & 0.28 & 0.32 & 0.40 & 0.15 & -0.20 & 0.47 & 0.01 & 0.95 \\
\hline
\end{tabular}

Ex= Exercise group; $C=$ Control group; $\mathrm{AST}=$ Aspartate aminotransferase; $\mathrm{ALT}=$ Alanine aminotransferase; $\mathrm{ALP}=\mathrm{Alkaline}$ phosphatase; $\mathrm{TGs}=$ Triglycerides; $\mathrm{TC}=$ Total cholesterol; $\mathrm{HDL}=$ High density lipoprotein; $\mathrm{LDL}=$ Low density lipoprotein; $* P<0.05$, significant correlation.

$R$; correlation coefficient, $P$; significant value.

weeks) in a study by Farzanegi et al, ${ }^{19}$ as well as the age ${ }^{27}$ and gender ${ }^{30}$ differences of subjects in other studies, ${ }^{29,30}$ may be possible reasons for the inconsistency with the results of our study.

Based on the World Health Organization (WHO), around $40 \%$ and $15 \%$ of women aged over 18 years are overweight and obese, respectively. ${ }^{32}$ On the other hand, the presence of obesity and overweight in Iranian women was estimated to be $30 \%$ and $20 \%$, respectively. ${ }^{33}$ In addition, our findings showed that hepatic enzymes are closely associated with changes in BMI, serum levels of lipid profiles, insulin and blood pressure, as well as the metabolic syndrome parameters such as age, weight, BMI, abdominal obesity (AO), waist circumference (WC), hip circumference (HC), and waist-to-hip ratio
(WHR). ${ }^{34}$ Since the AST and ALT have a half-life of approximately 17 and 42 hours respectively, ${ }^{12}$ it is possible that the persistent levels of hepatic enzymes after 24 hours following exercise in overweight PMWs may be related to the long-term half-life of AST and ALT ${ }^{12}$ and its likely to explain the high serum levels of hepatic enzymes in this study. It is well-known that aerobic exercise stimulates lipid oxidation and inhibits lipid synthesis in the liver which is activated by AMP-activated protein kinase (AMPK) pathway. ${ }^{31}$ In other words, the AMPK activated during exercise in skeletal muscle, liver, and adipose tissue ${ }^{31}$ has not been able to create physiological adaptations in the liver among overweight PMVs following 12 weeks of W-WJMIAEP-R exercise. On the other hand, it is likely that 12 weeks of W-WJMIAEP-R may lead to damage in 
hepatocytes, and as a result, elevated hepatic enzymes among overweight PMWs. ${ }^{31}$ On the other hand, the results of our study revealed that serum ALP increased in the Ex group after 12-week W-WJMIAEP-R intervention. Given that the effects of exercise on the hepatic enzymes are completely contradictory in related studies, ${ }^{1,17,18,26}$ it seems that the exact mechanisms are needed to be further evaluated regarding that whether the elevation in serum ALP is an exercise induced-positive physiological or a pathological adaptation.

On the other hand, due to the sedentary lifestyle and physically inactive status in overweight PMWs, it is likely that any intensity of aerobic exercise may cause additional pressure on body organs such as the liver and CVS. The studies have reported that regular exercise modulates the daily energy intake, lipid oxidation in the skeletal muscle and mitochondria, hepatocytes, and increases fat metabolism and redistribution in visceral fat tissues resulting in a decrease in free fatty acids (FFA) supply to the liver, a decrease for deposit of fat in the liver, and an increase in fat oxidation of the liver. ${ }^{35}$ However, it is possible that 12-week W-WJMIAEP-R intervention has not been able to stimulate fat oxidation in the liver, and this may require more than 12 weeks of $\mathrm{W}$-WJMIAEP-R intervention in overweight PMWs over 50 years old. The results of studies have shown that the aging process decreases daily physical activity level and basal metabolism rate (BMR) in the human, resulting in weight gain. Therefore, it is possible that low BMR and fat oxidation in PMW during 12 weeks of W-WJMIAEP-R intervention may be related to the paucity of fat metabolism in the Ex group compared to the $\mathrm{C}$ group. However, our study indicated that serum TC and LDL levels increased during 12 weeks in $\mathrm{C}$ group which probably was related to the aging process itself.

As mentioned above, overweight and physical inactivity lead to a decrease in daily energy intake, lipid oxidation in the skeletal muscle and mitochondria, decreased hepatocyte functions, altered fat metabolism, redistribution of fat stores in the body, decreased supply of FFA to the liver, and elevated fat oxidation in the liver. ${ }^{35}$ These events are risk factors for liver and CVS damage caused by the aging process among overweight PMWs over 50 years old. In fact, it is likely that these changes are caused by a reduction in estrogen and progesterone levels, physical inactivity, ${ }^{36}$ overweight, ${ }^{37}$ and accumulation of visceral fat during menopause. ${ }^{36,37}$ Moreover, the results of studies indicated that serum LDL as an atherogenic index of plasma (AIP) and serum HDL as an atherogenesis index are in the humans. ${ }^{38}$ Recently, epidemiological studies reported that low serum HDL level is related to an increase in serum LDL and risk factors of CVS, ${ }^{36}$ which may increase the AIP and reduce the atherogenesis index in the $\mathrm{C}$ group.

Additionally, the results of Pearson's correlation coefficient in our study showed that the ALP has a significant positive correlation with TC and LDL in the Ex group at baseline and week 12 post exercise. It seems that the ALP activity increases during menopause, which can affect serum levels of lipid profiles such as TC and LDL. Given that TC is one of the main sources for the synthesis of sex hormones, ${ }^{39,40}$ it is possible that changes in the serum levels of TC by estrogen and progesterone during menopause may be linked to the aging process. Since the transfer of cholesterol into the tissue is required for LDL production, the increase in TC can also affect serum levels of LDL during menopause. However, the dual mechanisms of increase in serum ALP during aerobic exercise in PMWs remain unclear and require further studies. In our study, there were significant positive correlations between serum levels of AST, TC and LDL after 12 weeks in the $\mathrm{C}$ group. It has been reported that the level of AST is directly related to the number of damaged cells, especially hepatocytes. ${ }^{7,9,11}$ Increase in the atherogenic factors such as LDL and TC can disrupt the liver function and CVS efficiency and facilitate the aging process. However, it is likely that increased serum levels of AST, TC, and LDL due to aging can be a risk factor for liver and CVS problems during menopause.

\section{Strengths and Limitations}

The use of a non-pharmacological prescription (i.e. W-WJMIAEP-R intervention) to prevent the liver disease or CVD in the context of menopause-induced changes in the hepatic enzymes and lipid profiles is the strength of our study. On the other hand, the low number of subjects is the main limitations of this study.

\section{Conclusion}

The findings of our study showed that 12-week W-WJMIEP-R with $65 \%$ to $70 \% \mathrm{HR}_{\max }$ did not affect hepatic enzymes and lipid profiles in overweight PMWs over 50 years old. However, the results of our study demonstrated that 12 weeks of W-WJMIEP-R intervention improved CRF through an increase in the $\mathrm{VO}_{2 \max }$ and WJTE and decrease in the SBP among overweight PMWs over 50 years old. In addition, a remarkable point in our study was that serum levels of ALP significantly increased after 12 weeks of W-WJMIEP-R intervention in the Ex group in overweight PMWs over 50 years old. In other words, it is likely that increased serum level of ALP, as well as increased CRF after 12 weeks of aerobic exercise intervention, can be as an exercise induced-positive physiological adaptation in the liver function among overweight PMWs over 50 years old.

\section{Ethical Approval}

This study was approved by the Ethics Committee of Allameh Tabataba'i University, Iran (Code: 1/3540).

\section{Competing Interests}

The authors of this study confirm that there was no 
competing interest.

\section{Acknowledgment}

The authors are very grateful to Allameh Tabataba'i University (due to supporting grant) and the women who participated in this study.

\section{References}

1. Smart NA, King N, McFarlane JR, Graham PL, Dieberg G. Effect of exercise training on liver function in adults who are overweight or exhibit fatty liver disease: a systematic review and meta-analysis. Br J Sports Med. 2018;52(13):834-843. doi:10.1136/bjsports-2016-096197

2. Gray B, Muhlhausler BS, Davies PS, Vitetta L. Liver enzymes but not free fatty acid levels predict markers of insulin sensitivity in overweight and obese, nondiabetic adults. Nutr Res. 2013;33:781-788. doi:10.1016/j. nutres.2013.07.019

3. Fleischman MW, Budoff M, Ifran Zeb DL, Foster T. NAFLD prevalence differs among hispanic subgroups: The multiethnic study of atherosclerosis. World J Gastroenterol. 2014;20:4987-4993. doi:10.3748/wjg.v20.i17.4987.

4. Lazo M, Clark JM. The epidemiology of nonalcoholic fatty liver disease: A global perspective. Semin Liver Dis. 2008;28:339-350. doi:10.1055/s-0028-1091978

5. Jamali R, Khonsari M, Merat S, Khoshnia M, Jafari E, Kalhori A. et al. Persistent alanine aminotransferase elevation among the general Iranian population: prevalence and causes. World J Gastroenterol. 2008;14:2867-2871. doi:10.3748/wjg.14.2867

6. Choi JW. Association between elevated serum hepatic enzyme activity and total body fat in obese humans. Ann Clin Lab Sci. 2003;33(3):257-264.

7. Chen S, Guo X, Yu S, Zhou Y, Li Z, Sun Y (2016). Metabolic syndrome and serum liver enzymes in the general Chinese population. Int J Environ Res Public Health. 2016;13(223):1-11. doi:10.3390/ijerph13020223

8. Villegas R, Xiang YB, Elasy T, et al. Liver enzymes, type 2 diabetes, and metabolic syndrome in middle-aged, urban Chinese men. Metab Syndr Relat Disord. 2011;9(4):305311. doi:10.1089/met.2011.0016

9. Hanley AJ, Williams K, Festa A, Wagenknecht LE, D’Agostino RB, Kempf J, et al. Elevations in markers of liver injury and risk of type 2 diabetes: The insulin resistance atherosclerosis study. Diabetes. 2004;53(10):2623-2632.

10. Najeebzadeh Q, Sameer AS, Azizand R, Hamid S. Association between elevated serum hepatic enzyme activity and total body fat in obese humans. Int J Current Research. 2015;7(4):14348-14352.

11. Sette HBC, Lopes EPA. Liver enzymes serum levels in patients with chronic kidney disease on hemodialysis: a comprehensive review. Clinics (Sao Paulo). 2014;69(4):271278. doi:10.6061/clinics/2014(04)09

12. Kim WR, Flamm SL, DiBisceglie AM, Bodenheimer HC. Serum activity of alanine aminotransferase (ALT) as an indicator of health and disease. Hepatology. 2008;47(4):1363-1370. doi:10.1002/hep.22109

13. Tartibian B, Sheikhlou Z, Malandish A, Rahmati-Yamchi M, Afsargharehbagh R. Effect of moderate-intensity aerobic training on alkaline phosphatase gene expression and serum markers of bone turnover in sedentary postmenopausal women. Tehran Univ Med J. 2017;74(10):723-734.

14. Yousefipoor P, Tadibi V, Behpoor N, Parnow A, Delbari M, Rashidi S. Effects of aerobic exercise on glucose control and cardiovascular risk factor in type 2 diabetes patients. Med J Mashhad Univ Med Sci. 2015;57(9):976-984. doi:10.22038/ mjms.2015.3882

15. Van-der-Windt D, Sud Y, Zhang H, Tsung A, Huang H. The effects of physical exercise on fatty liver disease. Gene Expr. 2018;18(2):89-101. doi:10.3727/10522161 7X15124844266408

16. Oh S, Han G, Kim B, Shoda J. Regular exercise as a secondary practical treatment for nonalcoholic fatty liver disease. Exer Med. 2018;2(4):1-7. doi:10.26644/em.2018.004

17. Hatami MT, Eftekhari E. The Efect of combined aerobic and resistance training on hepatic enzymes in males with nonalcoholic fatty liver. Biotech Health Sci. 2016;3(3):e36162. doi:10.17795/bhs-36162

18. Zar A, Hosseini S A, Homaion A. Effect of eight-week Aquagymnastic training on liver enzymes and lipid Profile of middle-aged women. Qom Univ Med Sci J. 2016;10(7):29-37.

19. Farzanegi P, Pouramin Z, Habibian M. Changes of liver trans-aminases after a period of selected aerobic training in postmenopausal women. Med Laboratory J. 2014;8(1):2329.

20. Brady CW. Liver disease in menopause. World J Gastroenterol. 2015 7;21(25):7613-7620. doi:10.3748/wjg. v21.i25.7613

21. Tajiri K, Shimizu Y. Liver physiology and liver diseases in the elderly. World J Gastroenterol. 2013;19:8459-8467. doi:10.3748/wjg.v19.i46.8459

22. Faul F, Erdfelder E, Lang AG, Buchner A. G*Power 3: a flexible statistical power analysis program for the social, behavioral, and biomedical sciences. Behav Res Methods. 2007;39(2):175-191. doi:10.3758/BF03193146

23. Sylvia LG, Bernstein EE, Hubbard JL, Keating L, Anderson EJ. Practical guide to measuring physical activity. J Acad Nutr Diet. 2014;114(2):199-208. doi:10.1016/j. jand.2013.09.018.

24. Bailey RL, Miller PE, Mitchell DC, Hartman TJ, Lawrence FR, Sempos CT, et al. dietary screening tool identifies nutritional risk in older adults. Am J Clin Nutr. 2009;90(1):177-183. doi:10.3945/ajcn.2008.27268.

25. Tartibian B, Afsargharehbagh R, Malandish A, Sheikhlou Z. Assessment of electrocardiogram indices in postmenopausal women: effects of aerobic exercise and detraining. Int J Basic Sci Med. 2018;3(1):3845. doi:10.15171/ijbms.2018.07

26. Hosseinikakhk A, Khaleghzadeh H, Nematy M, Hamedinia M. The effect of combined aerobic- resistance training on lipid profile and liver enzymes in patients with nonalcoholic fatty liver under nutrition diet. Sport Physiol. 2015;7(27):65-84.

27. Mohammadrahimi GR, Attarzadehosseini R. The effect of aerobic training and diet on lipid profile and liver enzymes in obese women with type II diabetes. Daneshvar Med. 2014;21(108):1-11.

28. Haghighi AH, Yaghoubi M, Hosseini-kakhk AR. The effect of eight weeks aerobic training and green Tea 
supplementation on body fat percentage and serum lipid profiles in obese and overweight women. Med J Mashhad Univ Med Sci. 2013;56(4):211-218. doi:10.22038/ mjms.2013.1757

29. Slentz CA, Bateman LA, Willis LH, et al. Effects of aerobic vs. resistance training on visceral and liver fat stores, liver enzymes, and insulin resistance by HOMA in overweight adults from STRRIDE AT/RT. Am J physiol. 2011;301(5):1033-1039. doi:10.1152/ajpendo.00291.2011

30. Shamsoddini A, Sobhani V, Ghamar Chehreh ME, Alavian SM, Zaree A. Effect of aerobic and resistance exercise training on liver enzymes and hepatic fat in Iranian men with nonalcoholic fatty liver disease. Hepat Mon. 2015;15(10):e31434. doi:10.5812/hepatmon.31434

31. Sadeghi S, Asad MR, Ferdosi MH. The effect of twelve weeks endurance training on liver enzymes levels in Iranian obese women. Research Sport Med Technol. 2017;15(13):49-60. [Persian].

32. Kelishadi R, Alikhani S, Delavari A, Alaedini F, Safaie A, Hojatzadeh E. Obesity and associated lifestyle behaviors in Iran: findings from the first national non-communicable disease risk factor surveillance survey. Public Health Nutr. 2008;11(03):246-251. doi:10.1017/S1368980007000262

33. Janghorbani M, Amini M, Willett WC, et al. First nationwide survey of prevalence of overweight, underweight, and abdominal obesity in Iranian adults. Obesity (Silver Spring). 2007;15(11):2797-2808. doi:10.1038/oby.2007.332

34. Bahari S, Faramarzi M, Azamian Jazi A, Cheragh Cheshm $M$. The effect of 8 weeks resistance training on resting level of liver inflammatory markers and insulin resistance of type 2 diabetic women. Armaghan Danesh. 2014;19(5):450-461.
[Persian].

35. Fathei M, Khairabadi S, Ramezani F, Hejazi K. The effects of eight weeks aerobic training, green tea supplementation and compound of them on serum liver enzymes and apolipoproteins in inactive overweight women. Med J Mashhad Univ Med Sci. 2016;59(2):114-123. doi:10.22038/ mjms.2016.7339. [Persian].

36. Tartibian B, Malandish A, AfsarGharehbagh R, Sheikhlou Z. Effects of 12 weeks of moderate-intensity aerobic exercise and 5 months detraining on cardiovascular biomarkers in inactive postmenopausal women. Razi J Med Sci. 2018;25(169):98-109.

37. Tartibian B, AfsarGharehbagh R, Malandish A, Sheikhlou Z. Correlation between blood pressure and vitamin $\mathrm{D}$, parathyroid hormone, calcium, and phosphorus in sedentary postmenopausal women. Tehran Univ Med J. 2016;74(8):554-561.

38. Orozco-Beltran D, Gil-Guillen VF, Redon J, MartinMoreno JM, Pallares-Carratala V, Navarro-Perez J. Lipid profile, cardiovascular disease and mortality in a Mediterranean high-risk population: The ESCARVALRISK study. PLoS One. 2017;12(10):e0186196. doi:10.1371/ journal.pone.0186196

39. Goedeke L, Fernandez-Hernando C. Regulation of cholesterol homeostasis. Cell Mol Life Sci. 2012;69(6):915930. doi:10.1007/s00018-011-0857-5

40. Moon JY, Choi MH, Kim J. Metabolic profiling of cholesterol and sex steroid hormones to monitor urological diseases. Endocr Relat Cancer. 2016;23(10):R455-R467. doi:10.1530/ERC-16-0285 